\title{
REVIEW
}

\section{Exercise rehabilitation following hospital discharge in survivors of critical illness: an integrative review}

\author{
Bronwen Connolly ${ }^{1,2}$, Linda Denehy ${ }^{3}$, Stephen Brett ${ }^{4}$, Doug Elliott ${ }^{5}$ and Nicholas Hart',2,6*
}

\begin{abstract}
Although clinical trials have shown benefit from early rehabilitation within the ICU, rehabilitation of patients following critical illness is increasingly acknowledged as an area of clinical importance. However, despite recommendations from published guidelines for rehabilitation to continue following hospital discharge, there is limited evidence to underpin practice during this intermediate stage of recovery. Those patients with ICU-acquired weakness on discharge from the ICU are most likely to benefit from ongoing rehabilitation. Despite this, screening based on strength alone may fail to account for the associated level of physical functioning, which may not correlate with muscle strength, nor address non-physical complications of critical illness. The aim of this review was to consider which patients are likely to require rehabilitation following critical illness and to perform an integrative review of the available evidence of content and nature of exercise rehabilitation programmes for survivors of critical illness following hospital discharge. Literature databases and clinical trials registries were searched using appropriate terms and groups of terms. Inclusion criteria specified the reporting of rehabilitation programmes for patients following critical illness post-hospital discharge. Ten items, including data from published studies and protocols from trial registries, were included. Because of the variability in study methodology and inadequate level of detail of reported exercise prescription, at present there can be no clear recommendations for clinical practice from this review. As this area of clinical practice remains in its relative infancy, further evidence is required both to identify which patients are most likely to benefit and to determine the optimum content and format of exercise rehabilitation programmes for patients following critical illness post-hospital discharge.
\end{abstract}

\section{Introduction}

Despite a greater prevalence of pre-existing co-morbidities in survivors, survival rates for critically ill patients admitted to the intensive care unit (ICU) have improved in recent years. Consequently, there is an increasing frequency of patients reporting both physical and psychological impairment with reduced healthrelated quality of life (HRQL) persisting long after the initial insult of the critical illness has resolved [1-11]. In many cases functional disability and psychological sequelae result in significant care-giver burden [12-14]. More recently, the rehabilitation requirements of patients surviving critical illness have been considered with the development of specific guidelines $[15,16]$. A seamless rehabilitation pathway from ICU admission to post-hospital

*Correspondence: nicholas.hart@gstt.nhs.uk

'Lane Fox Respiratory Unit, St Thomas' Hospital, Westminster Bridge Road, London SE1 7EH, UK

Full list of author information is available at the end of the article discharge has been advocated [16]. Although these guidelines have increased the profile of this important area of clinical practice, they lack evidence detailing the patients most likely to benefit and details of the exercise component of rehabilitation programmes. Clearly, the level or 'dosage' of an exercise prescription is essential in the same way a clinician advocates the use of a drug to treat a specific disease only after understanding the target population as well as the timing, dose and duration of the drug.

A significant body of evidence has emerged demonstrating that early mobilisation of patients, adopting clinical management algorithms, within the ICU is safe [17-19], effective and beneficial [20-31]. This is often characterised by a 'hierarchical progression' of techniques from passive movement to sitting, sit-to-stand, bed-tochair transfers, marching on the spot and ultimately walking [32-34]. In addition, the reliability and feasibility of mobility-based functional outcome measures has been demonstrated [30]. Comprehensive early rehabilitation, commencing from ICU admission and continuing throughout the hospital stay, has been shown to reduce 
ICU length of stay, improve functional performance status at hospital discharge and is more likely to result in discharge home [29].

The feasibility of specific ward-based rehabilitation intervention has been determined in two recent studies $[35,36]$ with further investigation planned of enhanced rehabilitation including physiotherapy and nutrition [36]. At this stage of recovery, the restoration of physical function aims to promote safe and effective hospital discharge. Beyond this stage, rehabilitation for ICU survivors has been inconsistent in delivery. Utilisation of post-hospital discharge ICU follow-up clinics has demonstrated mixed results [37], with the most recent study showing a lack of benefit [38]. Furthermore, only a small number of studies exist, both completed and ongoing, investigating the effect of rehabilitation programmes following discharge from hospital [35,37-45].

\section{Patients likely to benefit from exercise rehabilitation on hospital discharge}

Skeletal muscle wasting and weakness are common features in survivors of critical illness [46-49]. Commonly reported as ICU-acquired weakness (ICU-AW) [48], this is defined as weakness secondary to critical illness in the absence of any neurological or metabolic aetiology [50-52]. Risk factors for development of ICU-AW include severity of acute critical illness, level of systemic inflammatory response, length of ICU stay, duration of mechanical ventilation, prolonged immobility and duration of sedation [47,48,50,53-55]. Interestingly, the adverse effects of corticosteroids and neuromuscular blocking agents have recently been questioned following results of a randomised controlled trial investigating neuromuscular blocking agents in patients with acute lung injury [56-58]. There are no data reporting the effect of chronic cardiorespiratory disease, such as chronic obstructive pulmonary disease and chronic heart failure, as a risk factor for ICU-AW. Because these conditions demonstrate changes in both the size and type of skeletal muscle fibres $[59,60]$, it would be reasonable to assume critical illness could result in an acute-on-chronic muscle wasting condition in patients with such co-morbidities.

Long-term follow-up of patients following critical illness reveals deficits in both HRQL and exercise capacity evident for up to five years following the index admission [2,6,11,61]. Furthermore, this longstanding adverse effect on exercise capacity and HRQL in the presence of a restoration in lung function [11] implies that the long-term deficit is a consequence of ongoing skeletal muscle weakness, although this hypothesis has not yet been tested. These observations support physical rehabilitation therapy as a strategy to enhance exercise performance by improving muscular strength and endurance [62]. Exercise rehabilitation would therefore seem a rational approach in the post-critical illness population who continue to demonstrate ICU-AW at the time of discharge from critical care, although the most appropriate tool for diagnosing ICU-AW has yet to be determined. In a prospective uncontrolled study, exercise-based rehabilitation following hospital discharge was found to significantly improve exercise capacity and anxiety and depression in a cohort of 38 ICU survivors [44]. However, recent findings from a randomised controlled trial of a home-based physical rehabilitation programme showed no effect on HRQL and physical recovery [41]. One explanation for the lack of difference between intervention and control groups in this trial is that 'all comers' were included with no stratification of patients according to strength at point of study entry. These findings lend support to the rationale of directing future exercise-based rehabilitation trials following hospital discharge to patients with ICU-AW, rather than using mechanical ventilation hours or ICU length of stay as inclusion criteria.

\section{Screening patients by assessing muscle performance}

Assuming those patients with ongoing presentations of ICU-AW on discharge from the ICU would benefit from further rehabilitation, a screening tool is required during this stage of recovery. Manual muscle testing (MMT) offers a simple clinical test to determine muscle strength [52] and both the Medical Research Council (MRC) sum score [63] and handgrip dynamometry [46], as forms of MMT, have been used for this purpose. However, despite widespread use in clinical studies and practice, MMT in critically ill patients has limitations. Patients must be sufficiently awake and cognitively alert to perform the manoeuvres for each muscle assessment $[46,47,49]$. The clinician must also be confident that the patient is performing maximum volitional efforts. The ICU environment per se may not be conducive to such testing and extraneous conditions, such as pain, level of analgesia, level of sedation, the presence of arterial monitoring and venous access lines, can all affect patient compliance with the assessment resulting in poor reliability and reproducibility. In addition, the MRC sum score is dependent on the skill and experience of the clinician to deliver and assess the response of the patient both accurately and reliably. This results in differential sensitivity, or bias, across the levels of muscle strength, in particular for those not objectively quantified. Finally, standardisation of muscle testing position is essential for accurate and reliable results.

Despite the number of potential caveats associated with MMT to quantify muscle strength in critically ill patients, an MRC sum score of less than 48 (that is, $<4$ per muscle group tested) $[46,47,49,55,64-66]$ and a handgrip strength of $<11 \mathrm{~kg}$ for males and $<7 \mathrm{~kg}$ for females 
[46] is reported diagnostic of ICU-AW. More importantly, high levels of inter-rater reliability of the MRC sum score have been reported in stable patients in the recovery phase [67-69] and for handgrip dynamometry in awake and cooperative critically ill patients within the ICU [69]. Thus, although MMT has limitations in the early phase of critical illness, MMT could be used to facilitate screening patients for ICU-AW on discharge from critical care for enrolment to a post-critical care rehabilitation programme.

\section{Relationship between MRC sum score and functional performance}

The absence of a clear relationship between MRC sum score and functional performance remains a challenging factor. MRC sum score and handgrip dynamometry are static measures of strength and currently there are no data defining the relationship between ICU-AW and functional status. Future studies need to clearly define clinically significant weakness in this patient population. A linear relationship between ICU-AW and physical performance is unlikely as functional tests are complex motor tasks, whereas MMT measures assess individual muscle actions that do not represent muscle endurance, a property that represents the resistance of the muscle to fatigue. It remains a requirement to investigate the natural history of recovery of those patients with an MRC sum score $\geq 48$, which can be compared to that of weak patients (MRC sum score <48). If reduced physical performance is evident in clinically strong patients with an MRC sum score $\geq 48$, the entry criteria into a rehabilitation programme may need to be refined further to include a functional criterion. Currently only one posthospital discharge exercise rehabilitation study specifies MRC sum score $<48$ as a screening inclusion criterion [39] with 'normal physical function' a reported exclusion criterion for one other rehabilitation programme [43].

\section{Maximising activity within safety limits}

Several studies have established reasonable safety parameters for rehabilitating critically ill patients in the ICU environment $[15,18,19,24,27-30,70]$. Ward-based rehabilitation is similarly feasible with no reported adverse effects $[35,36]$. Patients commencing exercise rehabilitation following hospital discharge are at the next stage of physical recovery and, by inference, demonstrate greater clinical stability. Our own data have previously shown that early rehabilitation within one week of hospital discharge is both safe and beneficial following acute exacerbation of chronic obstructive pulmonary disease $[62,71]$, and the same is the case for post-critical illness patients [41,43]. All exercise rehabilitation programmes should include assessment of cardiac and respiratory risk factors before implementing an exercise programme to ensure patient safety, but this should not, however, detract from the goal of exercise rehabilitation, which requires a patient to achieve a sufficient work rate to produce a physiological training effect.

\section{Design of the review}

The aim of this integrative review was to report (1) the content of exercise rehabilitation programmes for survivors of critical illness on discharge from hospital with regard to timing of programmes and exercise prescription, and (2) considerations for relevant non-exercise components required in rehabilitation programmes. An integrative review method was adopted [72] to incorporate evidence obtained from studies with published data and protocols of ongoing trials listed on recognised trial databases. This approach enabled inclusion of a variety of study designs to inform knowledge on the topic, and is not restricted to randomised controlled trials. This approach was used as the body of evidence in this clinical area is limited, but evidence is growing as trials are ongoing.

\section{Search methods}

Published literature was identified through searches of relevant databases, including Pubmed, CINAHL, ScienceDirect, and the trial registration databases Clinical Trials.gov [73], Current Controlled Trials [74,75], INVOLVE [76] (part of the National Institute of Health Research [77]) and the Australian New Zealand Clinical Trials Registry [78]. The search terms used were 'rehabilitation following critical illness', 'exercise following critical illness', 'survivors of critical illness', 'follow-up after critical illness', 'physical recovery and critical illness', 'exercise and intensive care' and 'hospital discharge.' Further items were obtained through searching the reference lists of identified sources. Identified sources of evidence were only included if they (i) were reported from January 2000 to November 2011 and (ii) reported rehabilitation programmes for survivors of critical illness on discharge from hospital. Evidence was excluded if it solely pertained to rehabilitation of post-ICU patients whilst still in hospital. A total of only ten sources of evidence were identified that were eligible for inclusion, which are described in Table 1 [35,37-45].

\section{Prescription of exercise post-critical illness Timing of exercise programmes}

Early rehabilitation within one week of an acute exacerbation of chronic obstructive pulmonary disease is safe and effective, resulting in fewer hospital attendances [62] and improved exercise capacity [71]. In principle, this model can reasonably be applied in the post-critical care population. In the limited number of studies investigating post-hospital discharge exercise rehabilitation for patients following critical illness, programmes 


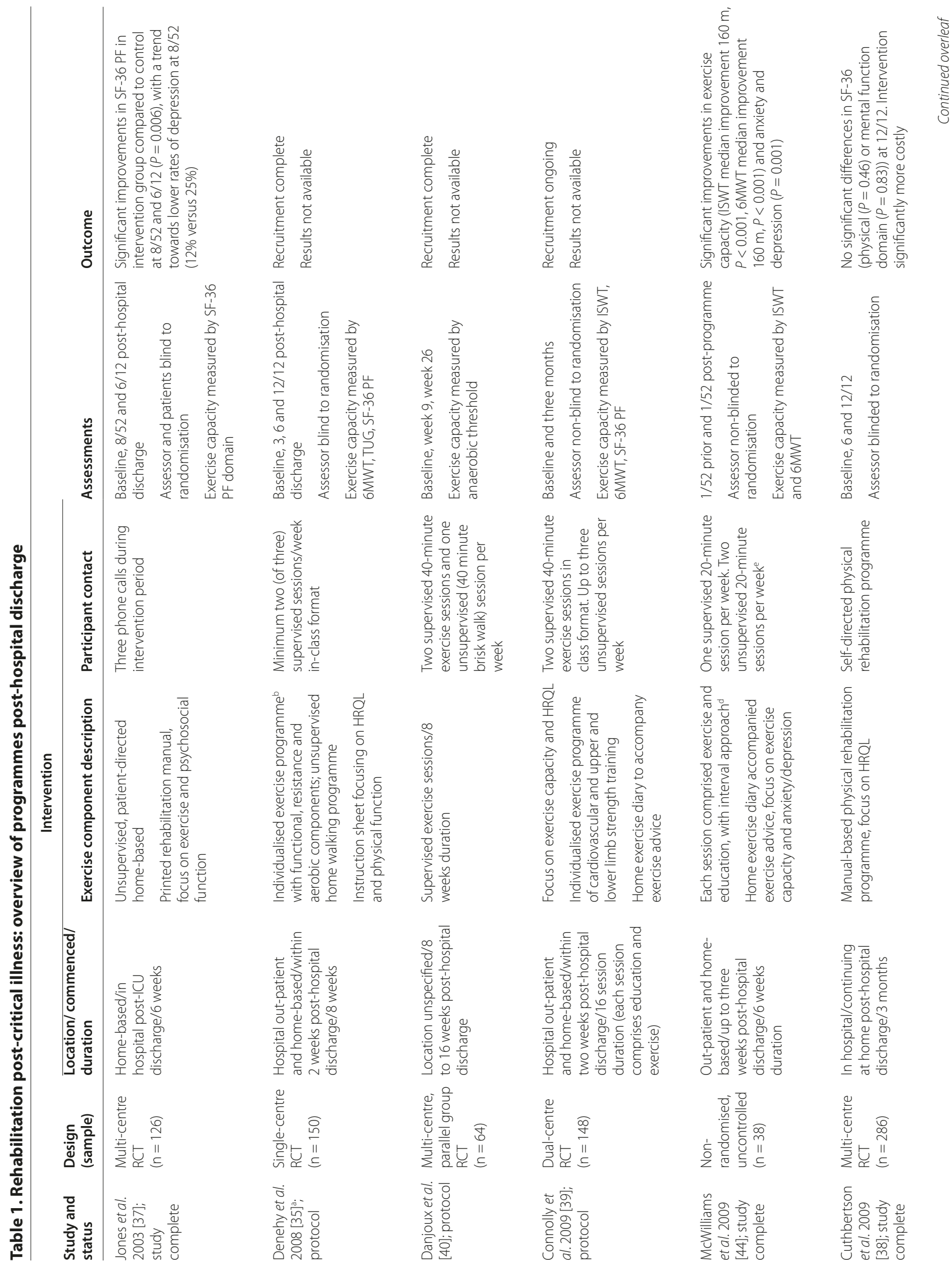




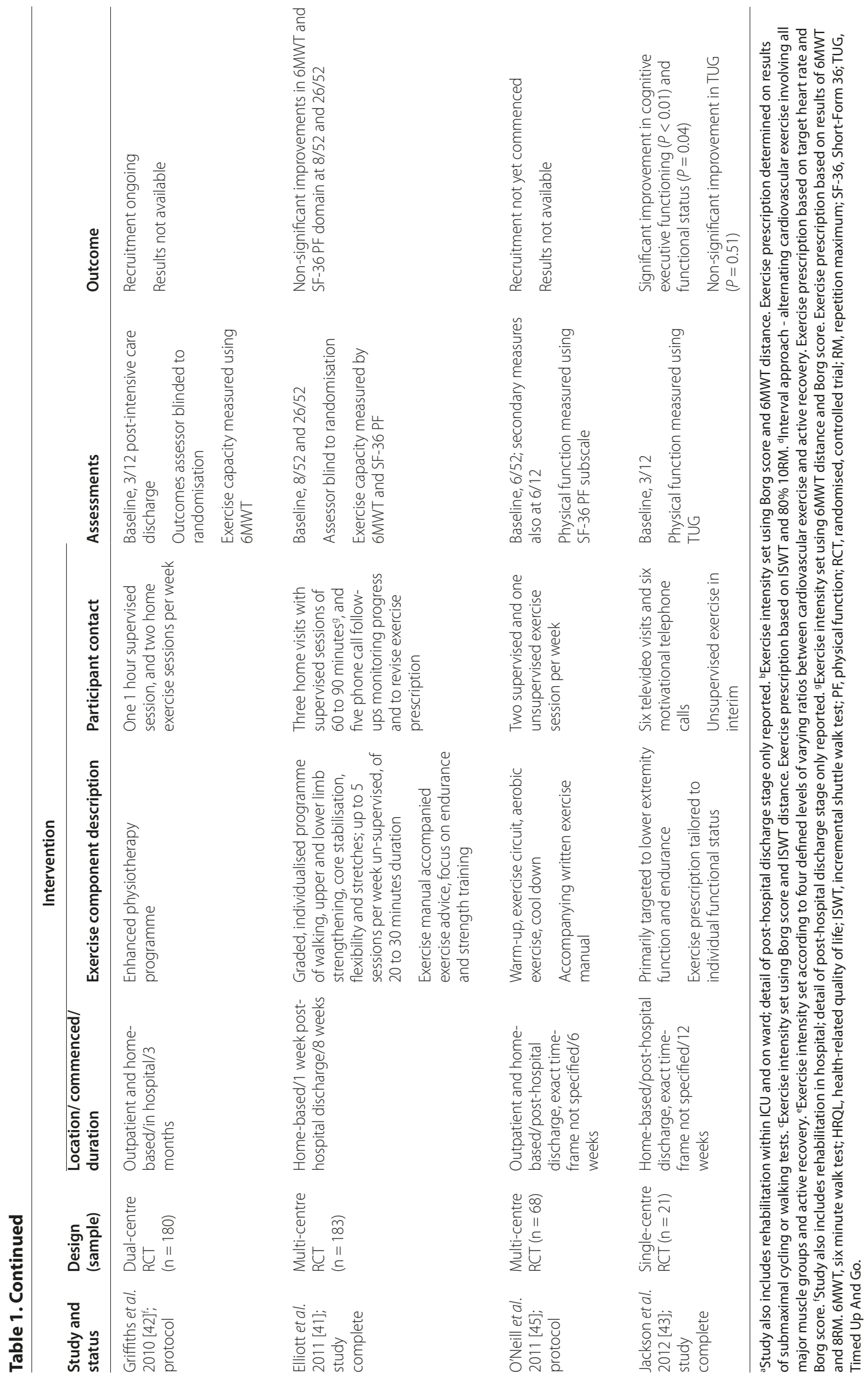


often commence within two weeks of discharge [35, $39,41]$ whilst the exact time-frame is not specified in two studies $[43,45]$. In many cases the rehabilitation programme commences during the in-patient stay $[35,37$, 38,42 ], implying a seamless continuation of the programme at hospital discharge. However, detail on the time-frame for commencing the post-hospital discharge stage of these rehabilitation programmes is only reported by one study [35]. One uncontrolled observational study had a delay of up to 3 weeks post-hospital discharge [44] and the baseline time point prior to intervention commencing in one study protocol is stated as 8 to 16 weeks following hospital discharge [40]. A significant delay in baseline assessment and initiation of exercisebased rehabilitation could reduce the effect size of any response to the rehabilitation, as any potential benefits may be masked by the extent of ongoing natural recovery.

For such studies, a control group is essential [35,37$43,45]$, which provides data on independent physical recovery following critical illness and also determines the true effect of exercise rehabilitation. To illustrate this point, significant improvements in exercise capacity following rehabilitation were demonstrated by McWilliams and colleagues [44]; however, a maximum time frame of three weeks between hospital discharge and initiation of the exercise rehabilitation programme and lack of a control group limited interpretation of the results. All the patients recruited were subject to physical recovery prerehabilitation, which would vary between individuals, and it is therefore impossible to separate the impact of the intervention from natural history of physical recovery. Further data are required to determine the optimum time for commencing rehabilitation programmes post-hospital discharge, although within two weeks appears common at present. Furthermore, there may be added value in commencing exercise rehabilitation on hospital discharge as an extension of an inhospital programme spanning ICU and the ward [35] to maximise the benefits gained to patients from familiarity and continuity with exercising.

\section{Type and dose of exercise prescription}

Lack of reported detail from some studies $[37,38,40$, 42,43 ], combined with variation in exercise prescription and study design, limits investigation of this area. Where greater detail is provided [35,39,41,44,45], a common feature is aerobic cardiovascular endurance activity, such as treadmill walking, ground walking or cycling. Initial prescriptions of exercise intensity utilise results of preliminary walking tests and cycle ergometry, and exercise intensity is monitored through the use of modified Borg scores and percentage of heart rate reserve. In many studies patients are instructed to exercise to between moderate and severe levels of breathlessness (Borg score 3 to 5) [35,39,41,44], although it is not yet confirmed that this level of exertion confers any benefit on functional or HRQL outcomes. Physiological measures such as Borg score and heart rate can be taught to patients to facilitate independent unsupervised exercise by providing clear limits for safely continuing exercise in the home environment at appropriate levels. Progression of exercise is determined using similar physiological measures and repeated exercise tests, although reference to performance of functional tests may also be of value. Strength exercises are also included in programmes $[35,39,41]$, most commonly employing the repetition maximum (RM) principle to guide initial resistance prescription. An assessment is made of the maximum weight a patient can lift over a set number of repetitions. Either a specific RM set is prescribed (for example, 8RM) [41] or a percentage of a specific RM set is used (for example, 80\% 10RM) [39]. Progression is based on reassessment of RM.

Two studies investigating detailed exercise rehabilitation programmes in patients post-critical illness on discharge from hospital have shown differences in their findings. Significant improvements were shown in exercise capacity in an uncontrolled study that adopted an interval style programme of cardiovascular activity and active recovery for 20-minute periods (Table 1). Different exercise intensities were achieved through altering the ratio of these components within the set time period. Heart rate reserve and Borg scores were used to determine exercise intensity and monitor status during exercise [44]. More recently, a randomised controlled trial found no significant differences between control and intervention groups that followed an 8-week, homebased, semi-supervised exercise programme of graded endurance and strength training of up to 60 to 90 minutes per session [41] (Table 1).

Furthermore, one recent study investigating a threearmed, home-based, semi-supervised rehabilitation protocol incorporating cognitive, physical and functional rehabilitation demonstrated non-significant improvements in physical function measured using the validated 'Timed Up And Go' measurement [43]. However, lack of reported detail of the exercise component limits further comment of these findings with regard to exercise prescription. Supervised exercise sessions allow clinicians to ensure patients are performing exercises correctly and safely, but may not be feasible in all situations, such as patients who live a long distance from the hospital. Some patients may also not wish to exercise in a group setting, but equally the psychological support from other patients may further encourage participation $[79,80]$. Data from pulmonary rehabilitation support supervised sessions as these provide enhanced clinical outcomes and patientcentred outcomes, such as increased exercise capacity 
and HRQL [81]. Unsupervised home exercise programmes are influenced not only by the motivation of the patient to engage and complete the exercise programme, but also the ability of the patients to correctly learn and perform the specific activities. An in-depth and detailed education package is required for this purpose. As adherence with documentation in home exercise diaries may be variable depending on the individual patient, pedometers [82] and accelerometers [83] could be used to encourage activity and provide objective monitoring and feedback on performance. These provide patients with more structured and objective home exercise programmes to supplement diary records.

\section{Non-exercise rehabilitation components}

Neurobehavioural outcomes, such as impaired cognitive function, are also recognised as consequences of critical illness [7]. Symptoms of anxiety and depression can be evident for up to two years following ICU discharge $[8,10]$ and other psychological issues are often present [84]. A recent stakeholder conference recommended the term 'post-intensive care syndrome' (PICS) to describe new or worsening problems in physical, cognitive, or mental health status persisting post-hospital discharge following a critical illness [85].

The impact of these impairments on HRQL for patients can be significant, resulting in disengagement from rehabilitation and a delay in physical recovery [10]. Furthermore, this disengagement could impact on motivation and the ability to perform volitional MMT, resulting, for example, in overestimation of ICU-AW on MRC sum score. Whilst the main goal of exercise rehabilitation programmes on discharge from hospital must be targeted at improving exercise capacity and physical activity, strategies to address psychological sequelae should also be included.

Jones and colleagues [37] demonstrated improvements, albeit non-significant, in symptoms of depression six months post-hospital discharge with the use of a self-help manual containing advice on psychological and psychosocial issues. Jackson and colleagues [43] acknowledged the potential benefit of cognitive rehabilitation to enhance functional outcome in post-critical illness patients using goal-management training to demonstrate significant improvements in cognitive executive functioning and activities of daily living. A logical approach, based on the prevalence and ranges in severity of psychological symptoms, would be to include a clinical psychologist in the post-critical illness rehabilitation team, although this needs to be tested. The established rehabilitation guidelines for cardiac and pulmonary rehabilitation programmes advocate a multidisciplinary approach to the delivery of rehabilitation [86-89]. In addition, cardiac and pulmonary rehabilitation guidelines recommend the inclusion of education sessions, albeit these are targeted at these specific disease groups. Only three of the recent clinical studies report education sessions with topics including benefits of exercise, stress management and nutritional advice $[37,39,44]$. However, the heterogeneity of the post-critical illness population, which may include patients with no significant pre-existing chronic disease, suggests caution should be used in applying standard education topics. Further investigation and determination of the most appropriate education and 'nonexercise' input required in rehabilitation programmes is urgently needed.

\section{Limitations of the review}

The aim of this integrative review was to focus on exercise rehabilitation programmes for patients following critical illness on discharge from hospital. It is important to highlight that only a very small number of sources were identified for inclusion, in itself a challenging factor for drawing clear conclusions from the evidence. Furthermore, only five studies reported published data $[37,38,41$, $43,44]$, whilst the remainder were available in protocol format only $[35,39,40,42,45]$. Of the published data, four were randomised controlled studies $[37,38,41,43]$, and one an observational study [44]. According to the GRADE system of classification of evidence sources [90], randomised controlled trial methodology automatically assumes the highest evidence grade, with observational studies low quality and other forms of evidence very low. Furthermore, evidence grading can be reduced if limitations are identified in the study [90]. In the context of this review, lack of reported detail on the exercise component of the rehabilitation programme $[37,38]$ could be considered a limitation influencing the strength of these studies. All five protocols reported ongoing randomised controlled trials. As none of these have published data at present, however, the advantages of the strong methodology employed are outweighed by lack of results on which to determine the success of these protocols.

\section{Conclusion and future directions}

Data are emerging that report the outcome of postcritical illness patients receiving exercise rehabilitation at the post-hospital discharge stage. Patients at risk of developing ICU-AW can be identified during the early stages of critical illness, and if clinically significant weakness persists on discharge from the ICU, are likely to be those who may benefit from ongoing rehabilitation in terms of exercise capacity, physical activity and HRQL. There are no data defining the relationship between peripheral muscle strength, functional capacity and physical activity in this recovery stage post-critical illness. Furthermore, at present the limited volume and 
variable quality of evidence available fails to provide substantive direction regarding optimum type and dose of exercise prescription or timing of rehabilitation required for this patient group. Future research, of robust methodology, needs to accurately address these issues and develop assessment tools that monitor the recovery of patients on which to substantiate rehabilitation needs. Ideally, a short battery of performance tests would encourage standardisation in practice between clinicians and facilitate comparison across future studies. Finally, the management of psychological consequences of critical illness also needs careful consideration and should be embedded in exercise-based rehabilitation programmes.

\section{Abbreviations}

6MWT, six minute walk test; HRQL, health-related quality of life; ICU-AW, ICU-acquired weakness; ISWT, incremental shuttle walk test; MMT, manual muscle testing; MRC, Medical Research Council; PF, Physical function; RCT, Randomised, controlled trial; RM, repetition maximum; SF-36, Short-Form 36: TUAG, Timed Up And Go.

\section{Competing interests}

The authors declare that they have no competing interests.

\section{Acknowledgements}

The authors acknowledge financial support from the Departments of Health via the National Institute for Health Research comprehensive Biomedical Research Centre award to Guy's \& St Thomas' NHS Foundation Trust (in partnership with King's College London and King's College Hospital NHS Foundation Trust) and to Imperial College Healthcare NHS Trust (in partnership with Imperial College London)

\section{Author details}

'Department of Asthma, Allergy and Respiratory Science, Division of Asthma, Allergy and Lung Biology, King's College London, London SE1 9RT, UK. 'uy's and St Thomas' NHS Foundation Trust and King's College London, National Institute of Health Research Comprehensive Biomedical Research Centre, London SE1 9RT, UK. ${ }^{3}$ Department of Physiotherapy, Melbourne School of Health Sciences, The University of Melbourne, Melbourne, 3010 Australia. ${ }^{4}$ Centre for Perioperative Medicine and Critical Care Research, Imperial College Healthcare NHS Trust, London W12 OHS, UK. ${ }^{5}$ Faculty of Nursing, Midwifery and Health, University of Technology, Sydney, Sydney, 2007 Australia. ' ${ }^{\circ}$ ane Fox Respiratory Unit, St Thomas' Hospital, Westminster Bridge Road, London SE1 7EH, UK

Published: 20 June 2012

\section{References}

1. Adamson $H$, Elliott D: Quality of life after a critical illness: a review of general ICU studies 1998-2003. Aust Crit Care 2005, 18:50-60.

2. Cheung AM, Tansey CM, Tomlinson G, Diaz-Granados N, Matté A, Barr A Mehta S, Mazer CD, Guest CB, Stewart TE, Al-Saidi F, Cooper AB, Cook D, Slutsky AS, Herridge MS: Two-year outcomes, health care use, and costs of survivors of acute respiratory distress syndrome. Am J Resp Crit Care Med 2006, 174:538-544

3. Dowdy D, Eid M, Sedrakyan A, Mendez-Tellez P, Pronovost P, Herridge M, Needham D: Quality of life in adult survivors of critical illness: a systematic review of the literature. Intensive Care Med 2005, 31:611-620

4. Elliott D: Measuring the health outcomes of general ICU patients: a systematic review of methods and findings. Aust Crit Care 1999, 12:132-140.

5. Fletcher SN, Kennedy DD, Ghosh IR, Misra VP, Kiff K, Coakley JH, Hinds CJ: Persistent neuromuscular and neurophysiologic abnormalities in longterm survivors of prolonged critical illness. Crit Care Med 2003, 31:1012-1016

6. Herridge MS, Cheung AM, Tansey CM, Matte-Martyn A, Diaz-Granados N,
Al-Saidi F, Cooper AB, Guest CB, Mazer CD, Mehta S, Stewart TE, Barr A, Cook D, Slutsky AS; Canadian Critical Care Trials Group: One-year outcomes in survivors of the acute respiratory distress syndrome. N Engl J Med 2003, 348:683-693.

7. Hopkins RO, Brett S: Chronic neurocognitive effects of critical illness. Curr Opin Crit Care 2005, 11:369-375.

8. Hopkins RO, Weaver LK, Collingridge D, Parkinson RB, Chan KJ, Orme JF Jr: Two-year cognitive, emotional, and quality-of-life outcomes in acute respiratory distress syndrome. Am J Respir Crit Care Med 2005, 171:340-347.

9. Oeyen SG, Vandijck DM, Benoit DD, Annemans L, Decruyenaere JM: Quality of life after intensive care: a systematic review of the literature. Crit Care Med 2010, 38:2386-2400.

10. Sukantarat K, Greer S, Brett S, Williamson R: Physical and psychological sequelae of critical illness. Br J Health Psychol 2007, 12:65-74.

11. Herridge MS, Tansey CM, Matté A, Tomlinson G, Diaz-Granados N, Cooper A, Guest CB, Mazer CD, Mehta S, Stewart TE, Kudlow P, Cook D, Slutsky AS, Cheung AM; Canadian Critical Care Trials Group: Functional disability 5 years after acute respiratory distress syndrome. NEngl J Med 2011, 364:1293-1304.

12. Chelluri L, Im KA, Belle SH, Schulz R, Rotondi AJ, Donahoe MP, Sirio CA, Mendelsohn AB, Pinsky MR: Long-term mortality and quality of life after prolonged mechanical ventilation. Crit Care Med 2004, 32:61-69.

13. de Miranda S, Pochard F, Chaize M, Megarbane B, Cuvelier A, Bele N, Gonzalez-Bermejo J, Aboab J, Lautrette A, Lemiale V, Roche N, Thirion M, Chevret S, Schlemmer B, Similowski T, Azoulay E: Postintensive care unit psychological burden in patients with chronic obstructive pulmonary disease and informal caregivers: A multicenter study. Crit Care Med 2011 39:112-118.

14. Kentish-Barnes N, Lemiale V, Chaize M, Pochard F, Azoulay E: Assessing burden in families of critical care patients. Crit Care Med 2009, 37(10 Suppl):S448-456

15. Gosselink R, Bott J, Johnson M, Dean E, Nava S, Norrenberg M, Schönhofer B, Stiller $\mathrm{K}$, van de Leur $\mathrm{H}$, Vincent J: Physiotherapy for adult patients with critical illness: recommendations of the European Respiratory Society and European Society of Intensive Care Medicine Task Force on Physiotherapy for Critically III Patients. Intensive Care Med 2008, 34:1188-1199.

16. NICE: Rehabilitation after Critical IIIness. NICE Clinical Guideline 83. London, UK: National Institute for Health and Clinical Excellence; 2009.

17. Hanekom S, Gosselink R, Dean E, van Aswegen H, Roos R, Ambrosino N, Louw $\mathrm{Q}$ : The development of a clinical management algorithm for early physical activity and mobilization of critically ill patients: synthesis of evidence and expert opinion and its translation into practice. Clin Rehabil 2011, 25:771-787.

18. Stiller K: Safety issues that should be considered when mobilizing critically ill patients. Crit Care Clin 2007, 23:35-53.

19. Stiller K, Phillips AC, Lambert P: The safety of mobilisation and its effect on haemodynamic and respiratory status of intensive care patients. Physiother Theory Pract 2004, 20:175-185.

20. Bailey P, Thomsen GE, Spuhler VJ, Blair R, Jewkes J, Bezdjian L, Veale K, Rodriquez L, Hopkins RO: Early activity is feasible and safe in respiratory failure patients. Crit Care Med 2007, 35:139-145.

21. Burtin C, Clerckx B, Robbeets C, Ferdinande P, Langer D, Troosters T, Hermans G, Decramer M, Gosselink R: Early exercise in critically ill patients enhances short-term functional recovery. Crit Care Med 2009, 37:2499-2505.

22. Chiang L-L, Wang L-Y, Wu C-P, Wu H-D, Wu Y-T: Effects of physical training on functional status in patients with prolonged mechanical ventilation. Phys Ther 2006, 86:1271-1281.

23. Kress JP, Pohlman AS, O'Connor MF, Hall JB: Daily interruption of sedative infusions in critically ill patients undergoing mechanical ventilation. NEngl J Med 2000, 342:1471-1477.

24. Morris PE, Goad A, Thompson C, Taylor K, Harry B, Passmore L, Ross A, Anderson L, Baker S, Sanchez M, Penley L, Howard A, Dixon L, Leach S, Small R, Hite RD, Haponik E: Early intensive care unit mobility therapy in the treatment of acute respiratory failure. Crit Care Med 2008, 36:2238-2243.

25. Morris PE, Herridge MS: Early intensive care unit mobility: future directions. Crit Care Clin 2007, 23:97-110.

26. Nava S: Rehabilitation of patients admitted to a respiratory intensive care unit. Arch Phys Med Rehabil 1998, 79:849-854.

27. Needham DM: Mobilizing patients in the intensive care unit: improving neuromuscular weakness and physical function. JAMA 2008, 300:1685-1690. 
28. Needham DM, Korupolu R, Zanni JM, Pradhan P, Colantuoni E, Palmer JB, Brower RG, Fan E: Early physical medicine and rehabilitation for patients with acute respiratory failure: a quality improvement project. Arch Phys Med Rehabil 2010, 91:536-542.

29. Schweickert WD, Pohlman MC, Pohlman AS, Nigos C, Pawlik AJ, Esbrook CL, Spears L, Miller M, Franczyk M, Deprizio D, Schmidt GA, Bowman A, Barr R, McCallister KE, Hall JB, Kress JP: Early physical and occupational therapy in mechanically ventilated, critically ill patients: a randomised controlled trial. Lancet 2009, 373:1874-1882.

30. Skinner EH, Berney S, Warrillow S, Denehy L: Development of a physical function outcome measure (PFIT) and a pilot exercise training protocol for use in intensive care. Crit Care Resusc 2009, 11:110-115.

31. Zanni JM, Korupolu R, Fan E, Pradhan P, Janjua K, Palmer JB, Brower RG, Needham DM: Rehabilitation therapy and outcomes in acute respiratory failure: An observational pilot project. J Crit Care 2010, 25:254-262.

32. Baker C, Mansfield L: Physical rehabilitation following critical illness. JICS 2008, 9:166-169

33. Skinner EH, Berney S, Warrillow S, Denehy L: Rehabilitation and exercise prescription in Australian intensive care units. Physiotherapy 2008, 94:220-229.

34. Stiller K, Phillips A: Safety aspects of mobilising acutely ill patients. Physiother Theory Pract 2003, 19:239-257.

35. Denehy L, Berney S, Skinner EH, Edbrooke L, Warrillow S, Hawthorne G, Morris ME: Evaluation of exercise rehabilitation for survivors of intensive care: protocol for a single blind randomised controlled trial. Open Crit Care Med J 2008, 1:39-47.

36. Salisbury L, Merriweather J, Walsh T: The development and feasibility of a ward-based physiotherapy and nutritional rehabilitation package for people experiencing critical illness. Clin Rehabil 2010, 24:489-500.

37. Jones C, Skirrow P, Griffiths R, Humphris G, Ingleby S, Eddleston J, Waldmann C, Gager M: Rehabilitation after critical illness: a randomized, controlled trial. Crit Care Med 2003, 31:2456-2461.

38. Cuthbertson BH, Rattray J, Campbell MK, Gager M, Roughton S, Smith A, Hull A, Breeman S, Norrie J, Jenkinson D, Hernández R, Johnston M, Wilson E, Waldmann C; PRaCTICaL study group: The PRaCTICaL study of nurse led, intensive care follow-up programmes for improving long term outcomes from critical illness: a pragmatic randomised controlled trial. BMJ 2009, 339:b3723.

39. Connolly B, Hart N: Clinicaltrials.gov: Rehabilitation Following Critical IIIness NCT00976807 [http://clinicaltrials.gov/ct2/show/NCT00976807]

40. Danjoux G: Current Controlled Trials: The Effect of Exercise on Patient Fitness and Quality of Life After Intensive Care Admission ISRCTN65176374. [http://www.controlled-trials.com/ISRCTN65176374]

41. Elliott D, McKinley S, Alison J, Aitken L, King M, Leslie G, Kenny P, Taylor P, Foley $\mathrm{R}$, Burmeister $\mathrm{E}$ : Health-related quality of life and physical recovery after a critical illness: a multi-centre randomised controlled trial of a home-based physical rehabilitation program. Crit Care 2011, 15:R142.

42. Griffiths R: Clinicaltrials.gov: Rehabilitating Muscle After Intensive Care (REMAIC) NCT01063738. [http://clinicaltrials.gov/ct2/show/NCT01063738]

43. Jackson JCP, Ely EWMDMPH, Morey MCP, Anderson VMMA, Denne LBMSW, Clune JMD, Siebert CSMS, Archer KRP, Torres RMS, Janz DMD, Schiro EBA, Jones JBA, Shintani AKP, Levine BP, Pun BTMSN, Thompson JMA, Brummel NEMD, Hoenig HMD: Cognitive and physical rehabilitation of intensive care unit survivors: Results of the return randomized controlled pilot investigation. Crit Care Med 2012, 40:1088-1097.

44. McWilliams DJ, Atkinson D, Carter A, Foëx BA, Benington S, Conway DH: Feasibility and impact of a structured, exercise-based rehabilitation programme for intensive care survivors. Physiother Theory Pract 2009, 25:566-571

45. O'Neill B: Clinicaltrials.gov: Exercise After Intensive Care Unit: a Randomised Controlled Trial (REVIVE) NCT01463579 [http://clinicaltrials. gov/ct2/show/NCT01463579]

46. Ali NA, O'Brien JM Jr, Hoffmann SP, Phillips G, Garland A, Finley JC, Almoosa K, Hejal R, Wolf KM, Lemeshow S, Connors AF Jr, Marsh CB; Midwest Critical Care Consortium: Acquired weakness, handgrip strength, and mortality in critically ill patients. Am J Resp Crit Care Med 2008, 178:261-268.

47. De Jonghe B, Sharshar T, Lefaucheur JP, Authier FJ, Durand-Zaleski I, Boussarsar M, Cerf C, Renaud E, Mesrati F, Carlet J, Raphaël JC, Outin H, Bastuji-Garin S; Groupe de Réflexion et d'Etude des Neuromyopathies en Réanimation: Paresis acquired in the intensive care unit. JAMA 2002 288:2859-2867.
48. Schweickert WD, Hall J: ICU-acquired weakness. Chest 2007, 131:1541-1549.

49. Sharshar T, Bastuji-Garin S, Stevens RD, Durand MC, Malissin I, Rodriguez P, Cerf C, Outin H, De Jonghe B: Presence and severity of intensive care unitacquired paresis at time of awakening are associated with increased intensive care unit and hospital mortality. Crit Care Med 2009, 37:3047-3053.

50. Griffiths RD, Hall JB: Exploring intensive care unit-acquired weakness. Preface. Crit Care Med 2009, 37(10 Suppl):S295.

51. Stevens RD, Marshall SA, Cornblath DR, Hoke A, Needham DM, de Jonghe B, Ali NA, Sharshar T: A framework for diagnosing and classifying intensive care unit-acquired weakness. Crit Care Med 2009, 37(10 Suppl):S299-308.

52. Vincent $\mathrm{J}$, , Norrenberg M: Intensive care unit-acquired weakness: framing the topic. Crit Care Med 2009, 37(10 Suppl):S296-298.

53. de Jonghe B, Lacherade J-C, Sharshar T, Outin H: Intensive care unitacquired weakness: Risk factors and prevention. Crit Care Med 2009, 37:S309-S315

54. de Letter M-ACJ, Schmitz PIM, Visser LH, Verheul FAM, Schellens RLLA, Op de Coul DAW, van der Meche FGM: Risk factors for the development of polyneuropathy and myopathy in critically ill patients. Crit Care Med 2001, 29:2281-2286

55. Nanas S, Kritikos K, Angelopoulos E, Siafaka A, Tsikriki S, Poriazi M, Kanaloupit D, Kontogeorgi M, Pratikaki M, Zervakis D, Routsi C, Roussos C: Predisposing factors for critical illness polyneuromyopathy in a multidisciplinary intensive care unit. Acta Neurol Scand 2008, 118:175-181.

56. Papazian L, Forel JM, Gacouin A, Penot-Ragon C, Perrin G, Loundou A, Jaber S, Arnal JM, Perez D, Seghboyan JM, Constantin JM, Courant P, Lefrant JY, Guérin C, Prat G, Morange S, Roch A; ACURASYS Study Investigators: Neuromuscular blockers in early acute respiratory distress syndrome. N Engl J Med 2010, 363:1107-1116.

57. Papazian L, Forel JM, Roch A: Neuromuscular blockers and ARDS. N Engl J Med 2010, 363:2562-2564

58. Puthucheary Z, Rawal J, Ratnayake G, Harridge S, Montgomery H, Hart N: Neuromuscular blockade and skeletal muscle weakness in critically ill patients: time to re-think the evidence? Am J Respir Crit Care Med 2012, 185:911-917.

59. Gosker HR, Kubat B, Schaart G, van der Vusse GJ, Wouters EFM, Schols AMWJ: Myopathological features in skeletal muscle of patients with chronic obstructive pulmonary disease. Eur Respir J 2003, 22:280-285.

60. Mancini D, Walter G, Reichek N, Lenkinski R, McCully K, Mullen J, Wilson J: Contribution of skeletal muscle atrophy to exercise intolerance and altered muscle metabolism in heart failure. Circulation 1992, 85:1364-1373.

61. Unroe M, Kahn JM, Carson SS, Govert JA, Martinu T, Sathy SJ, Clay AS, Chia J, Gray A, Tulsky JA, Cox CE: One-year trajectories of care and resource utilization for recipients of prolonged mechanical ventilation: a cohort study. Ann Intern Med 2010, 153:167-175.

62. Seymour JM, Moore L, Jolley CJ, Ward K, Creasey J, Steier JS, Yung B, Man WD Hart N, Polkey MI, Moxham J: Outpatient pulmonary rehabilitation following acute exacerbations of COPD. Thorax 2010, 65:423-428.

63. Kleyweg RP, van der Meche FG, Schmitz PI: Interobserver agreement in the assessment of muscle strength and functional abilities in Guillain-Barre syndrome. Muscle Nerve 1991, 14:1103-1109.

64. De Jonghe B, Bastuji-Garin S, Durand M-C, Malissin I, Rodrigues P, Cerf C, Outin H, Sharshar T, for Groupe de Reflexion et d'Etude des Neuromyopathies En R: Respiratory weakness is associated with limb weakness and delayed weaning in critical illness. Crit Care Med 2007, 35:2007-2015.

65. De Jonghe B, Bastuji-Garin S, Sharshar T, Outin H, Brochard L: Does ICUacquired paresis lengthen weaning from mechanical ventilation? Intensive Care Med 2004, 30:1117-1121.

66. Lefaucheur J-P, Nordine T, Rodriguez P, Brochard L: Origin of ICU acquired paresis determined by direct muscle stimulation. J Neurol Neurosurg Psychiatry 2006, 77:500-506

67. Fan E, Ciesla N, Truong A, Bhoopathi V, Zeger S, Needham D: Inter-rater reliability of manual muscle strength testing in ICU survivors and simulated patients. Intensive Care Med 2010, 36:1038-1043.

68. Hough C, Lieu B, Caldwell E: Manual muscle strength testing of critically ill patients: feasibility and interobserver agreement. Crit Care 2011, 15:R43.

69. Vanpee G, Segers J, Van Mechelen H, Wouters P, Van der Berghe G, Hermans G, Gosselink R: The interobserver agreement of handheld dynamometry for muscle strength assessment in critically ill patients. Crit Care Med 2011, 39:1929-1934

70. Kress JP: Clinical trials of early mobilization of critically ill patients. Crit Care Med 2009, 37(10 Suppl):S442-447. 
71. Man WD-C, Polkey MI, Donaldson N, Gray BJ, Moxham J: Community pulmonary rehabilitation after hospitalisation for acute exacerbations of chronic obstructive pulmonary disease: randomised controlled study. BMJ 2004, 329:1209.

72. Whittemore $R$, Knafl K: The integrative review: updated methodology. J Adv Nurs 2005, 52:546-553.

73. Clinical Trials.gov [http://www.clinicaltrials.gov/]

74. Current Controlled Trials [http://www.controlled-trials.com/]

75. ISRCTN Register [http://isrctn.org/]

76. INVOLVE [http://www.invo.org.uk]

77. National Institute of Health Research [http://www.nihr.ac.uk/]

78. Australian New Zealand Clinical Trials Registry [http://www.anzctr.org.au/]

79. O'Shea SD, Taylor NF, Paratz JD: But watch out for the weather: factors affecting adherence to progressive resistance exercise for persons with COPD. J Cardiopulm Rehabil 2007, 27:166-174.

80. Wilson JS, O'Neill B, Reilly J, MacMahon J, Bradley JM: Education in pulmonary rehabilitation: the patient's perspective. Arch Phys Med Rehabil 2007, 88:1704-1709.

81. Troosters T, Gosselink R, Janssens W, Decramer M: Exercise training and pulmonary rehabilitation: new insights and remaining challenges. Eur Respir Rev 2010, 19:24-29.

82. Moore R, Berlowitz D, Denehy L, Jackson B, McDonald CF: Comparison of pedometer and activity diary for measurement of physical activity in chronic obstructive pulmonary disease. J Cardpulm Rehabil 2009, 29:57-61.

83. Pitta F, Troosters T, Probst VS, Spruit MA, Decramer M, Gosselink R: Quantifying physical activity in daily life with questionnaires and motion sensors in COPD. Eur Respir J 2006, 27:1040-1055.

84. Jones C, Griffiths RD: Long-term outcome from critical illness. Anaesthesia Intensive Care Med 2006, 7:155-156.
85. Needham DM, Davidson J, Cohen H, Hopkins RO, Weinert C, Wunsch H, Zawistowski C, Bemis-Dougherty A, Berney SC, Bienvenu OJ, Brady SL, Brodsky MB, Denehy L, Elliott D, Flatley C, Harabin AL, Jones C, Louis D, Meltzer W, Muldoon SR, Palmer JB, Perme C, Robinson M, Schmidt DM, Scruth E, Spill GR, Storey CP, Render M, Votto J, Harvey MA: Improving long-term outcomes after discharge from intensive care unit: Report from a stakeholders' conference. Crit Care Med 2012, 40:502-509.

86. BTS statement. Pulmonary rehabilitation. British Thoracic Society Standards of Care Subcommittee on Pulmonary Rehabilitation. Thorax 2001, 56:827-834.

87. BACR: Standards and Core Components for Cardiac Rehabilitation. British Association for Cardiac Rehabilitation; 2007.

88. Ries AL, Bauldoff GS, Carlin BW, Casaburi R, Emery CF, Mahler DA, Make B, Rochester CL, ZuWallack R, Herrerias C: Pulmonary rehabilitation: joint ACCP/AACVPR evidence-based clinical practice guidelines. Chest 2007, 131 (5 suppl):4S-42S

89. SIGN: Cardiac Rehabilitation: a National Clinical Guideline. Scottish Intercollegiate Guidelines Network; 2002.

90. Guyatt GH, Oxman AD, Kunz R, Vist GE, Falck-Ytter Y, Schünemann HJ: What is "quality of evidence" and why is it important to clinicians? BMJ 2008 336:995-998.

doi:10.1186/cc11219

Cite this article as: Connolly B, et al:: Exercise rehabilitation following hospital discharge in survivors of critical illness: an integrative review. Critical Care 2012, 16:2?? 